\title{
A Novel Prediction Model for Bloodstream Infections in Hepatobiliary-Pancreatic Surgery Patients
}

\author{
Po-Sheng Yang ${ }^{1,2}$ (1) $\cdot$ Chang-Pan Liu ${ }^{3} \cdot$ Yi-Chiung Hsu ${ }^{4} \cdot$ Chuen-Fei Chen ${ }^{1} \cdot$ Chi-Chan Lee $^{2} \cdot$ \\ Shih-Ping Cheng ${ }^{1,2}$
}

Published online: 2 January 2019

(C) The Author(s) 2019

\begin{abstract}
Background Bloodstream infections (BSI) are an important source of postoperative mortality in hepatobiliarypancreatic surgery (HBPS) patients, and no prediction model has been analyzed before.

Methods Using big data from the electronic medical records of the administrative and culture databases of MacKay Memorial Hospital, we identified the potential risk factors for community-acquired and healthcare-associated BSI and mortality of patients who received HBPS. Subsequently, we analyzed the microorganisms' profiles and antimicrobial susceptibility patterns for these BSI.

Results BSI were found in 6.3\% patients (349 of 5513 HBPS patients), and hospital mortality was $1.48 \%$ (82 of 5513). Dividing patients into low-, intermediate-, and high-risk groups on the basis of sex, age, status of comorbidity (renal failure, peptic ulcer disease, fluid and electrolyte disorders, and acute cholecystitis), a predictive BSI risk score model was developed. According to this model, BSI risk ranged from 1.43\% to $11.95 \%$; AUROC to predict BSI risk was 0.72 (95\% CI 0.69-0.75). From this retrospective study, Enterobacteriaceae were the most common microorganisms that were isolated from BSI. For both community-acquired and healthcare-associated BSI, imipenem and colistin are the most successful.

Conclusion This novel model can be useful to predict who is at risk of BSI after HBPS, and new prophylactic protocols for these patients are needed.
\end{abstract}

Electronic supplementary material The online version of this article (https://doi.org/10.1007/s00268-018-04903-x) contains supplementary material, which is available to authorized users.

\section{Po-Sheng Yang}

psyang@mmc.edu.tw

1 Department of Medicine, MacKay Medical College, No. 46, Sec. 3, Zhongzheng Rd., Sanzhi Dist., New Taipei City 252, Taiwan

2 Department of General Surgery, MacKay Memorial Hospital, Taipei, Taiwan

3 Department of Infection Control Center, MacKay Memorial Hospital, Taipei, Taiwan

4 Department of Biomedical Sciences and Engineering, National Central University, Taoyuan, Taiwan

\section{Introduction}

Hepatobiliary and pancreatic diseases frequently require major surgical procedures involving the liver, bile duct, pancreas, duodenum, and other organs. Several studies have revealed that morbidity and mortality rates have improved for these procedures recently [1-5]. However, postoperative mortality and morbidity rates after hepatobiliary-pancreatic surgery (HBPS) remain high, even at high-volume centers. According to Japanese nationwide surveys, postoperative mortality rates for hepatectomy and pancreaticoduodenectomy are $2.6 \%$ and $2.8-3.5 \%$, while overall morbidity rates are $14.5 \%$ and $40 \%$, respectively $[6,7]$. Infectious complications are one of the main causes of death. Surgical-site infection rates after HBPS were 
reported to be about 9.9-23\% [8]. Postoperative infectious complication rates for liver resection were reported as 15.6-34\% [9-11], which adversely affect oncologic outcomes for hepatocellular carcinoma patients [11], potentially resulting in hepatic failure and death. Furthermore, the infections in these HBPS patients are associated with high morbidity and mortality [12].

Recently, more research has been done using electronic medical record data from administrative databases. This big data can be analyzed for epidemiology, quality of care, pharmacovigilance, and surgical outcome [13]. Some studies employed the Elixhauser Comorbidity Index-30 comorbid conditions [14] defined from US administrative health data that have been proven to provide valid prognosis for poor patient outcomes, including increased inhospital mortality, medical cost, and length of stay-to categorize their data. Some liver disease studies have furthermore shown that the Elixhauser index performed better than the Charlson Comorbidity Index in analyzing administrative health data [15].

However, past studies have rarely studied bloodstream infections (BSI) in HBPS patients by jointly analyzing risk factors according to the Elixhauser comorbidities, microorganisms' profiles, and antimicrobial susceptibility patterns. This is most likely because the technology required to analyze such vast amounts of data-thousands of cases were examined in this novel study-was not as widely available until now. Our interdisciplinary study combined bioinformatics, computer science, and clinical medicine. We attempted to identify the potential risk factors for community-acquired and healthcare-associated BSI and to analyze the mortality rates for patients who received HBPS, according to the electronic medical records from MacKay Memorial Hospital's large administrative and culture databases. The results of this study will enable physicians to better employ preventative and responsive measures against BSI for high-risk patients (as determined by their comorbidities) who receive HBPS.

\section{Materials and methods}

\section{Study design and patients}

Approval from the Ethics Committee of the MacKay Memorial Hospital Institutional Review Board was obtained for this study (14MMHIS227) before the data were obtained from electronic hospital records. The investigation was designed as a retrospective cohort study of all patients (aged $\geq 20$ ) who received surgery for hepatobiliary and pancreatic diseases, excluding transplantations, from January 2008 to December 2014 at MacKay Memorial Hospital. These HBPS patients received prophylactic antibiotic with $1 \mathrm{~g}$ cefazolin $30 \mathrm{~min}$ before operation except those patients who had received therapeutic antibiotics. The clinical data coded ICD-9-CM were collected from records in the hospital's admissions database. These included admission and discharge codes for diagnosis, procedures, complications, and discharge status. All episodes of BSI were collected from culture database in Infection Control Center Department of MacKay Memorial Hospital, and each case was categorized as communityacquired or healthcare-associated. Programs written in Python 2.7 with package NumPy, SciPy, and CSV were used to analyze the data. We first grouped the data by the HBPS patients' diagnosis codes according to the Elixhauser comorbidity. We then calculated the BSI frequency as well as mortality rate for the each of the comorbidities on the Elixhauser Comorbidity Index. The risk factors for BSI and mortality of HBPS were analyzed according to the items on the Elixhauser Comorbidity Index. Then, we tested various microorganisms isolated from communityacquired and healthcare-associated BSI and compared their antimicrobial susceptibilities.

All episodes of BSI after HBPS at MacKay Memorial Hospital were reported and followed by infectious disease physicians and the Infection Control Center Department, who then advised antibiotic treatment (excluding first-line antibiotics). The antimicrobial susceptibilities for different drugs of microorganisms isolated from the two categories of BSI were then analyzed.

\section{Definitions}

The underlying diseases of comorbidity were defined based on standard criteria. Comorbidity data were grouped according to the Elixhauser Comorbidity Index using ICD9-CM codes from the administrative database [14] for each patient. The HBPS cases were selected from those with ICD-9-CM procedure codes. We defined liver surgery to include ICD-9-CM 50.00, 50.12, 50.21, 50.22, 50.29, 50.3, 50.61 , and 50.99 (transplantation was excluded); biliary surgery to include all 51.xx codes except 51.01, 51.02, $51.1 \mathrm{x}, 51.8 \mathrm{x}$, and 51.90; pancreatic surgery to include all 52.xx codes except 52.01, 52.1x, 52.8x, and 52.9x (listed as supplement Table). Significant bloodstream infections were defined as those with one or more blood cultures positive for one or more primary pathogens, and demonstrating clinically apparent signs and symptoms of sepsis [16]. For analysis purposes, we divided the origin of BSI into two categories: community-acquired and healthcareassociated, in accordance with the classic United States Center for Disease Control and Prevention (CDC) criteria [17]. Healthcare-associated BSI was defined if any of the following criteria was met [18]: $>48 \mathrm{~h}$ of hospital admission in the previous 90 days, receipt of hemodialysis, 
Table 1 Analysis of bloodstream infections (BSI) with different comorbidities in patients with hepatobiliary and pancreatic surgery

\begin{tabular}{|c|c|c|c|c|c|}
\hline Comorbidity & $\begin{array}{l}\text { No BSI patients } \\
(N=5164,93.7 \%)\end{array}$ & $\begin{array}{l}\text { BSI patients } \\
(N=349,6.3 \%)\end{array}$ & Univariate $P$ & $\begin{array}{l}\text { Multivariate OR } \\
(95 \% \text { CI })\end{array}$ & $P$ \\
\hline Congestive heart failure & $78(1.5 \%)$ & $16(4.6 \%)$ & $<0.05$ & $1.64(0.89-2.83)$ & 0.09 \\
\hline Cardiac arrhythmias & $78(1.5 \%)$ & $10(2.9 \%)$ & 0.07 & & NS \\
\hline Valvular disease & $56(1.1 \%)$ & $8(2.3 \%)$ & 0.06 & & NS \\
\hline Peripheral vascular disorders & $6(0.1 \%)$ & $2(0.6 \%)$ & 0.08 & & NS \\
\hline Hypertension & $1111(21.5 \%)$ & $77(22.1 \%)$ & 0.79 & & NS \\
\hline Paralysis & $1(0.02 \%)$ & 0 & 1 & & NS \\
\hline Other neurological disorders & $13(0.3 \%)$ & $1(0.28 \%)$ & 0.60 & & NS \\
\hline Chronic pulmonary disease & $82(1.6 \%)$ & $10(2.9 \%)$ & 0.08 & & NS \\
\hline Diabetes (uncomplicated) & $770(14.9 \%)$ & $84(24.1 \%)$ & $<0.05$ & $1.28(0.97-1.67)$ & 0.07 \\
\hline Diabetes (complicated) & $27(0.5 \%)$ & 0 & 0.41 & & NS \\
\hline Hypothyroidism & $9(0.2 \%)$ & 0 & 1 & & NS \\
\hline Renal failure & $65(1.3 \%)$ & $16(4.6 \%)$ & $<0.05$ & $2.5(1.35-4.37)$ & 0.002 \\
\hline Liver disease & $646(12.5 \%)$ & $43(12.3 \%)$ & 1 & & NS \\
\hline Peptic ulcer disease (excluding bleeding) & $566(11 \%)$ & $58(16.6 \%)$ & $<0.05$ & $1.5(1.1-2.02)$ & 0.008 \\
\hline AIDS & $2(0.04 \%)$ & 0 & 1 & & NS \\
\hline Lymphoma & $3(0.06 \%)$ & $1(0.28 \%)$ & 0.23 & & NS \\
\hline Metastatic cancer & $347(6.7 \%)$ & $35(10 \%)$ & $<0.05$ & $1.37(0.92-1.97)$ & 0.10 \\
\hline Solid tumor without metastasis & $1030(19.9 \%)$ & $85(24.4 \%)$ & 0.053 & & NS \\
\hline Rheumatoid arthritis and collagen vascular diseases & $26(0.5 \%)$ & 0 & 0.41 & & NS \\
\hline Coagulopathy & $36(0.7 \%)$ & $5(1.4 \%)$ & 0.18 & & NS \\
\hline Weight loss & $2(0.04 \%)$ & $4(1.1 \%)$ & $<0.05$ & $28.5(5.1-217.94)$ & $<0.001$ \\
\hline Fluid and electrolyte disorders & $51(0.98 \%)$ & $14(4.0 \%)$ & $<0.05$ & $2.87(1.46-5.28)$ & 0.001 \\
\hline Anemia due to Blood loss & $11(0.2 \%)$ & $2(0.6 \%)$ & 0.20 & & NS \\
\hline Deficiency anemia & $84(1.6 \%)$ & $10(2.9 \%)$ & 0.09 & & NS \\
\hline Alcohol abuse & $19(0.36 \%)$ & $1(0.38 \%)$ & 1 & & NS \\
\hline Drug abuse & $2(0.04 \%)$ & 0 & 1 & & NS \\
\hline Psychoses & $35(0.68 \%)$ & $2(0.57 \%)$ & 1 & & NS \\
\hline Depression & $9(0.17 \%)$ & $1(0.28 \%)$ & 0.48 & & NS \\
\hline Age $>65$ years & $1309(25.3 \%)$ & $193(55.3 \%)$ & $<0.05$ & $3.17(2.52-4)$ & $<0.001$ \\
\hline
\end{tabular}

$N S$ not significant

receipt of intravenous medication or home wound care in the previous 30 days, and residence in a nursing home or long-term care facility. Any other BSI episodes were defined as community-acquired. We defined mortality to include all causes of death during admission for all cases with positive bloodstream cultures after HBPS.

\section{Microbiologic studies}

Two sets of two 8-10 mL blood samples (an aerobic and anaerobic sample in each set) were taken $30 \mathrm{~min}$ apart for all HBPS patients with a fever $>38.5^{\circ} \mathrm{C}$ or when bacteremia was clinically suspected. The blood samples were processed by the BACTEC 9240 system (Becton-Dickinson Microbiology Systems, Sparks, Md.), with an incubation period of 5 days. Isolates were identified by standard techniques. Minimum inhibitory concentration (MICs) of antimicrobial agents against Gram-negative bacilli were determined by the broth microdilution method using custom-made cassettes $\left(\right.$ VITEK $^{\circledR} 2$ system, Hazelwood, MO, USA) containing 15 such agents commonly used to treat bacteremia. The agents tested included ampicillin-sulbactam, amikacin, ceftriaxone, ceftazidime, cefotaxime, cefoxitin, cefepime, ertapenem, imipenem, piperacillin-tazobactam, levofloxacin, colistin, tigecycline, gentamycin, and ciprofloxacin. The MICs of five other antimicrobial agents countering Gram-positive cocci were determined using the same method. The agents tested included gentamycin, ciprofloxacin, vancomycin, oxacillin, and linezolid. Susceptibility was determined according to 
Table 2 Analysis of mortality with different comorbidities in patients with hepatobiliary and pancreatic surgery

\begin{tabular}{|c|c|c|c|c|c|}
\hline Comorbidity & $\begin{array}{l}\text { Alive } \\
(N=5431,85.1 \%)\end{array}$ & $\begin{array}{l}\text { Dead } \\
(N=82,14.9 \%)\end{array}$ & Univariate $P$ & $\begin{array}{l}\text { Multivariate OR } \\
(95 \% \mathrm{CI})\end{array}$ & $P$ \\
\hline Congestive heart failure & $83(1.53 \%)$ & $11(13.4 \%)$ & $<0.001$ & $5.81(2.58-12.1)$ & $<0.001$ \\
\hline Cardiac arrhythmias & $85(1.57 \%)$ & $3(3.7 \%)$ & 0.14 & & NS \\
\hline Valvular disease & $62(1.14 \%)$ & $2(2.4 \%)$ & 0.25 & & NS \\
\hline Peripheral vascular disorders & $7(0.012 \%)$ & $1(1.2 \%)$ & 0.11 & & NS \\
\hline Hypertension & $1180(21.7 \%)$ & $8(9.8 \%)$ & 0.007 & $0.32(0.14-0.66)$ & 0.004 \\
\hline Paralysis & $1(0.02 \%)$ & 0 & 1 & & NS \\
\hline Other neurological disorders & $14(0.26 \%)$ & 0 & 1 & & NS \\
\hline Chronic pulmonary disease & $90(1.7 \%)$ & $2(2.4 \%)$ & 0.40 & & NS \\
\hline Diabetes (uncomplicated) & $844(15.5 \%)$ & $10(12.2 \%)$ & 0.54 & & NS \\
\hline Diabetes (complicated) & $26(0.48 \%)$ & $1(1.2 \%)$ & 0.33 & & NS \\
\hline Hypothyroidism & $9(0.17 \%)$ & 0 & 1 & & NS \\
\hline Renal failure & $66(1.2 \%)$ & $15(18.3 \%)$ & $<0.001$ & $7.59(3.66-15)$ & $<0.001$ \\
\hline Liver disease & $669(12.3 \%)$ & $20(24.4 \%)$ & 0.003 & $1.35(0.7-2.49)$ & 0.35 \\
\hline Peptic ulcer disease (excluding bleeding) & $618(11.4 \%)$ & $6(7.3 \%)$ & 0.30 & & NS \\
\hline AIDS & $2(0.04 \%)$ & 0 & 1 & & NS \\
\hline Lymphoma & $3(0.06 \%)$ & $1(1.2 \%)$ & 0.06 & & NS \\
\hline Metastatic cancer & $368(6.8 \%)$ & $14(17.1 \%)$ & 0.001 & $1.26(0.58-2.63)$ & 0.55 \\
\hline Solid tumor without metastasis & $1074(19.8 \%)$ & $41(50 \%)$ & $<0.001$ & $2.77(1.51-4.98)$ & $<0.001$ \\
\hline Rheumatoid arthritis and collagen vascular diseases & $26(0.5 \%)$ & 0 & 1 & & NS \\
\hline Coagulopathy & $33(0.61 \%)$ & $8(9.6 \%)$ & $<0.001$ & $9.16(3.35-22.81)$ & $<0.001$ \\
\hline Weight loss & $6(0.11 \%)$ & 0 & 1 & & NS \\
\hline Fluid and electrolyte disorders & $63(1.2 \%)$ & $2(2.4 \%)$ & 0.25 & & NS \\
\hline Anemia due to blood loss & $13(0.24 \%)$ & 0 & 1 & & NS \\
\hline Deficiency anemia & $91(1.8 \%)$ & $3(3.7 \%)$ & 0.16 & & NS \\
\hline Alcohol abuse & $20(0.37 \%)$ & 0 & 1 & & NS \\
\hline Drug abuse & $2(0.04 \%)$ & 0 & 1 & & NS \\
\hline Psychoses & $37(0.68 \%)$ & 0 & 1 & & NS \\
\hline Depression & $10(0.18 \%)$ & 0 & 1 & & NS \\
\hline Age $>65$ years & $1452(26.7 \%)$ & $50(61.0 \%)$ & $<0.001$ & $2.54(1.54-4.21)$ & $<0.001$ \\
\hline Bloodstream infection & $322(5.9 \%)$ & $27(32.9 \%)$ & $<0.001$ & $5.13(3-8.6)$ & $<0.001$ \\
\hline
\end{tabular}

NS not significant

Clinical and Laboratory Standards Institute (CLSI) breakpoints [19] and European Committee on Antimicrobial Susceptibility Testing breakpoint [20].

\section{Statistical analysis}

Demographic and clinical variables, defined as comorbidity according to the Elixhauser Comorbidity Index using ICD-9CM codes from the administrative database, were evaluated for their effect on BSI and in-hospital mortality, using Chisquare test and multivariable logistic regression. Statistical significance was defined as a two-tailed $P$ value $<0.05$. For analysis purposes, we considered BSI-related and BSI- unrelated mortality (within same hospitalization of bloodstream infection) together. The Chi-square test and logistic regression were accomplished using $\mathrm{R}$ version 3.2.1.

We developed a risk score model to predict the risk of bloodstream infection. Risk factors according to the Elixhauser Comorbidity Index and diseases codes using ICD-9$\mathrm{CM}$ were included in the risk score model. Development of the risk score had three steps. First, a multiple logistic regression model was used to estimate the $\beta$ regression coefficient, $P$ value, and odds ratio and its $95 \%$ CI for each of the selected risk predictors. Second, the odds ratio in the multiple logistic regression model of each risk predictor was divided by the male-to-female odds ratio, and then, it 
Table 3 Logistic regression model by multivariate analysis of the risk of bloodstream infections and corresponding risk score

\begin{tabular}{|c|c|c|c|c|}
\hline Variable & OR $(95 \% \mathrm{CI})$ & $\beta$ Coefficient & $P$ value & Risk score \\
\hline \multicolumn{5}{|l|}{ Sex } \\
\hline Female & 1.00 & & & 0 \\
\hline Male & $1.60(1.28-2.01)$ & 0.4713 & $<.0001$ & 1 \\
\hline \multicolumn{5}{|c|}{ Age (years) } \\
\hline$\leqq 55$ & 1.00 & & & 0 \\
\hline $55^{+}$to 65 & $2.75(1.99-3.80)$ & 1.01 & $<.0001$ & 2 \\
\hline$>65$ & $5.19(3.90-6.90)$ & 1.65 & $<.0001$ & 3 \\
\hline \multicolumn{5}{|c|}{ Renal failure } \\
\hline No & 1.00 & & & 0 \\
\hline Yes & $2.23(1.25-3.98)$ & 0.80 & 0.0069 & 1 \\
\hline \multicolumn{5}{|c|}{ Peptic ulcer disease } \\
\hline No & 1.00 & & & 0 \\
\hline Yes & $1.42(1.05-1.92)$ & 0.35 & 0.0236 & 1 \\
\hline \multicolumn{5}{|c|}{ Fluid and electrolyte disorders } \\
\hline No & 1.00 & & & 0 \\
\hline Yes & $2.92(1.56-5.48)$ & 1.07 & 0.0008 & 2 \\
\hline \multicolumn{5}{|c|}{ Acute cholecystitis } \\
\hline No & 1.00 & & & 0 \\
\hline Yes & $1.93(1.27-2.95)$ & 0.66 & 0.0023 & 1 \\
\hline
\end{tabular}

Table 4 Cumulative risk score and associated risk of bloodstream infections

\begin{tabular}{llccc}
\hline Risk group & $\begin{array}{l}\text { Index } \\
\text { score }\end{array}$ & Patients & $\begin{array}{l}\text { Bloodstream } \\
\text { infections } n(\%)\end{array}$ & $P$ value \\
\hline Low & 0 & 1464 & $21(1.43)$ & $<.0001$ \\
Intermediate & $1-2$ & 1924 & $74(3.85)$ & \\
High & $\geqq 3$ & 2125 & $254(11.95)$ & \\
\hline
\end{tabular}

was rounded into an integer value to generate the risk score. Third, a cumulative risk score was then developed for the risk of bloodstream infection.

\section{Results}

\section{Prognostic determinants of BSI}

A total of 5513 patients who received surgery for hepatobiliary and pancreatic diseases (excluding transplantation patients) from January 2008 to December 2014 in MacKay Memorial Hospital were included in this study. Their ages ranged from 20 to 99 years old (mean 55.4 years): 2573 were male, and 2940 were female.

BSI were found in 349 patients (6.3\% of 5513 patients). Congestive heart failure, uncomplicated diabetes mellitus, renal failure, peptic ulcer disease, metastatic cancer, weight loss, fluid and electrolyte disorders (according to Elixhauser Comorbidity Index, the fluid and electrolyte disorders include the following ICD-9 codes: 276.0-276.9, hyperosmolality and/or hypernatremia, hyposmolality and/ or hyponatremia, acidosis, alkalosis, mixed acid-base balance disorder, volume depletion, fluid overload, hyperpotassemia, hypopotassemia, electrolyte and fluid disorders) and an age greater than 65 years were associated with BSI by univariate analysis (Table 1). Multivariate analysis found that renal failure, peptic ulcer disease, weight loss, fluid and electrolyte disorders, and an age greater than 65 years were associated with BSI.

\section{Mortality}

In total, 82 of the 5513 patients $(1.48 \%)$ in this study died in hospital (Table 2). Univariate analysis revealed that mortality was associated with congestive heart failure, hypertension, renal failure, liver disease, metastatic cancer, solid tumor without metastasis, coagulopathy, an age greater than 65 years and BSI. Multivariate analysis revealed that the risk factors for mortality were congestive heart failure, hypertension, renal failure, solid tumor without metastasis, coagulopathy, an age greater than 65 years and BSI.

\section{Developing a risk score model to predict the risk of bloodstream infection in HBPS patients}

Table 3 shows $\beta$ regression coefficient, $\mathrm{p}$ value, odds ratio, and its $95 \% \mathrm{CI}$ for each of the selected risk predictors using the multiple logistic model and corresponding risk score. Risk factors were sex, age, renal failure, peptic ulcer disease, fluid and electrolyte disorders and acute cholecystitis. The cumulative risk score was defined as the sum of the number of risk factors present with each risk factor receiving a value of 1 , except for age and fluid and electrolyte disorders, which was scored as 2 for patients aged 56-65 years, 3 for patients aged $>65$ years, and 2 for patients with fluid and electrolyte disorders. Risk groups were defined by comparing the odds ratios of bloodstream infection in patients with different cumulative risk scores and combining categories with similar odds ratios. We plotted receiver operating curve (ROC) and calculated the area under receiver operating curve (AUROC) to show the discrimination capability for bloodstream infection risk. Table 4 shows the cumulative risk score and associated risk of bloodstream infections. Patients were grouped as low risk (score 0 ), intermediate risk (score 1 or 2), or high risk (score $\geqq 3$ ). For example, a male patient (risk score $=1$ ), aged 60 years $(2)$, who had renal failure (1), but did not have peptic ulcer disease (0), fluid and electrolyte disorders (0) and acute cholecystitis (0) would have a 
Table 5 Microorganisms in community-acquired and the antimicrobial susceptibility of different drugs for bloodstream infection in patients with hepatobiliary and pancreatic surgery

\begin{tabular}{|c|c|c|c|c|c|}
\hline \multirow[t]{2}{*}{ Microorganisms (no.) } & \multicolumn{5}{|l|}{ Drugs } \\
\hline & 3rd cephalosporin $n(\%)$ & 4th cephalosporin $n(\%)$ & Imipenem $n(\%)$ & Quinolone $n(\%)$ & Colistin $n(\%)$ \\
\hline Gram-negative (239) & $195(81.6 \%)$ & $220(92.1 \%)$ & $228(98.7 \%)^{*}$ & $169(70.7 \%)$ & $220(100 \%)^{*}$ \\
\hline Enterobacteriaceae (230) & $187(81.3 \%)$ & $211(91.7 \%)$ & $227(98.7 \%)$ & $160(69.6 \%)$ & $220(100 \%)^{*}$ \\
\hline Escherichia coli (141) & $114(80.9 \%)$ & $130(92.2 \%)$ & $139(98.6 \%)$ & $96(68.1 \%)$ & $141(100 \%)$ \\
\hline Klebsiella pneumoniae (68) & $56(82.4 \%)$ & $60(88.2 \%)$ & $67(98.5 \%)$ & $43(63.2 \%)$ & $68(100 \%)$ \\
\hline Klebsiella oxytoca (5) & $4(80 \%)$ & $5(100 \%)$ & $5(100 \%)$ & $5(100 \%)$ & $5(100 \%)$ \\
\hline Morganella morganii (5) & $4(80 \%)$ & $5(100 \%)$ & $5(100 \%)$ & $5(100 \%)$ & NT \\
\hline Proteus vulgaris (3) & $2(66.7 \%)$ & $3(100 \%)$ & $3(100 \%)$ & $3(100 \%)$ & NT \\
\hline Enterobacter aerogenes (2) & $2(100 \%)$ & $2(100 \%)$ & $2(100 \%)$ & $2(100 \%)$ & $2(100 \%)$ \\
\hline Enterobacter cloacae (2) & $1(50 \%)$ & $2(100 \%)$ & $2(100 \%)$ & $2(100 \%)$ & $2(100 \%)$ \\
\hline Proteus mirabilis (2) & $2(100 \%)$ & $2(100 \%)$ & $2(100 \%)$ & $2(100 \%)$ & NT \\
\hline Salmonella spp. (2) & $2(100 \%)$ & $2(100 \%)$ & $2(100 \%)$ & $2(100 \%)$ & $2(100 \%)$ \\
\hline Non-Enterobacteriaceae (9) & $8(88.9 \%)$ & $9(100 \%)$ & $1(100 \%)$ & $9(100 \%)$ & \\
\hline Aeromonas spp. (8) & $8(100 \%)$ & $8(100 \%)$ & NT & $8(100 \%)$ & NT \\
\hline Pseudomonas aeruginosa (1) & 0 & $1(100 \%)$ & $1(100 \%)$ & $1(100 \%)$ & NT \\
\hline Gram-positive & Oxacillin & Vancomycin & Linezolid & Gentamycin-H & \\
\hline Staphylococcus aureus (7) & $4(57.1 \%)$ & $7(100 \%)$ & $7(100 \%)$ & NT & \\
\hline Enterococcus faecium (6) & NT & $5(83.3 \%)$ & $6(100 \%)$ & $6(100 \%)$ & \\
\hline Enterococcus faecalis (5) & NT & $5(100 \%)$ & $5(100 \%)$ & $5(100 \%)$ & \\
\hline Enterococcus casseliflavus (3) & NT & $1(33.3 \%)$ & $3(100 \%)$ & $3(100 \%)$ & \\
\hline Enterococcus gallinarum (3) & NT & $3(100 \%)$ & $3(100 \%)$ & $3(100 \%)$ & \\
\hline
\end{tabular}

3rd cephalosporin: ceftazidime and ceftriaxone; 4th cephalosporin: cefpirome

$N T$ not test

${ }^{a}$ Excluded the NT episodes

cumulative risk score of 4 (in the high-risk group), with a projected bloodstream infection risk of $11.95 \%$. We plotted ROC with AUROC of 0.72 (95\% CI 0.69-0.75) showing a good discrimination capability for bloodstream infection risk (Supplementary Figure 1).

\section{Microbiological data}

The most common microorganism is E. coli, followed by Klebsiella pneumoniae, Aeromonas hydrophila/caviae, Staphylococcus aureus (MRSA), alpha-Streptococcus species, and Enterococcus spp. The most common microorganism in healthcare-associated episodes is Candida albicans, followed by E. coli, Acinetobacter baumannii, $K$. pneumoniae, and Enterococcus spp.

\section{Antimicrobial susceptibilities of different drugs for microorganisms in community-acquired BSI in HBPS patients}

The antimicrobial susceptibilities to different drugs for microorganisms in community-acquired BSI in patients with HBPS are shown in Table 5. The rate of susceptibility of Gram-negative microorganisms to third-generation cephalosporin is $81.6 \%(195 / 239), 92.1 \%(220 / 239)$ to fourth-generation cephalosporin, $98.7 \%$ (228/231) to carbapenem (imipenem), only $70.7 \%$ to quinolone, and $100 \%$ to colistin. For Gram-positive microorganisms, we found that Staphylococcus aureus isolates were less susceptible to oxacillin $(57.1 \%, 4 / 7)$ compared to other microorganisms.

\section{Antimicrobial susceptibilities of different drugs for microorganisms in healthcare-associated BSI in HBPS patients}

The antimicrobial susceptibilities to different drugs for microorganisms in health-associated BSI in patients with HBPS are shown in Table 6. The rate of susceptibility of Gram-negative microorganisms to third-generation cephalosporin is only $36.8 \%$ (25/68), 55.9\% (38/68) to fourthgeneration cephalosporin, $65.2 \%(43 / 66)$ to carbapenem (imipenem), only $47.1 \%$ (32/68) to quinolone, and $90.9 \%$ (60/66) to colistin. We found that $E$. coli isolates were less susceptible to third- and fourth-generation cephalosporins, quinolone, and colistin than isolates of other Enterobacteriaceae in this study. For non-Enterobacteriaceae Gram- 
Table 6 Microorganisms in healthcare-associated bacteremia and the antimicrobial susceptibility rate of different drugs for bloodstream infection in patients with hepatobiliary and pancreatic surgery

\begin{tabular}{|c|c|c|c|c|c|}
\hline \multirow[t]{2}{*}{ Microorganisms (no.) } & \multicolumn{5}{|l|}{ Drugs } \\
\hline & 3rd cephalosporin $n(\%)$ & 4th cephalosporin $n(\%)$ & Imipenem $n(\%)$ & Quinolone $n(\%)$ & Colistin $n(\%)$ \\
\hline Gram-negative (68) & $25(36.7 \%)$ & $38(55.9 \%)$ & $43(65.2 \%)^{\mathrm{a}}$ & $32(47.1 \%)$ & $60(90.9 \%)^{\mathrm{a}}$ \\
\hline Enterobacteriaceae (42) & $22(52.4 \%)$ & $31(73.8 \%)$ & $36(85.7 \%)$ & $25(59.5 \%)$ & $36(85.7 \%)$ \\
\hline Escherichia coli (16) & $6(37.5 \%)$ & $8(50 \%)$ & $13(81.3 \%)$ & $7(43.8 \%)$ & $11(68.8 \%)$ \\
\hline Klebsiella pneumoniae (11) & $8(72.7 \%)$ & $10(90.9 \%)$ & $10(90.9 \%)$ & $8(72.7 \%)$ & $10(90.9 \%)$ \\
\hline Enterobacter cloacae (7) & $4(57.1 \%)$ & $6(85.7 \%)$ & $6(85.7 \%)$ & $4(57.1 \%)$ & $7(100 \%)$ \\
\hline Enterobacter aerogenes (4) & $2(50 \%)$ & $4(100 \%)$ & $4(100 \%)$ & $3(75 \%)$ & $4(100 \%)$ \\
\hline Serratia marcescens (4) & $2(50 \%)$ & $3(75 \%)$ & $3(75 \%)$ & $3(75 \%)$ & $4(100 \%)$ \\
\hline Non-Enterobacteriaceae (26) & $3(11.5 \%)$ & $7(26.9 \%)$ & $7(29.2 \%)^{\mathrm{a}}$ & $7(26.9 \%)$ & $24(100 \%)^{\mathrm{a}}$ \\
\hline Acinetobacter baumannii (13) & 0 & 0 & 0 & 0 & $13(100 \%)$ \\
\hline Pseudomonas aeruginosa (6) & 0 & $3(50 \%)$ & $5(83.3 \%)$ & $5(83.3 \%)$ & $6(100 \%)$ \\
\hline Acinetobacter lwoffii (3) & $1(33.3 \%)$ & $1(33.3 \%)$ & $1(33.3 \%)$ & $1(33.3 \%)$ & $3(100 \%)$ \\
\hline Acinetobacter junii (2) & $1(50 \%)$ & $1(50 \%)$ & $1(50 \%)$ & 0 & $2(100 \%)$ \\
\hline Aeromonas spp. (2) & $1(50 \%)$ & $2(100 \%)$ & NT & $1(50 \%)$ & NT \\
\hline Gram-positive & Oxacillin & Vancomycin & Linezolid & Gentamycin-H & \\
\hline Enterococcus faecalis (8) & NT & $5(62.5 \%)$ & $8(100 \%)$ & $4(50 \%)$ & \\
\hline Enterococcus faecium (7) & NT & $5(71.4 \%)$ & $7(100 \%)$ & $3(42.9 \%)$ & \\
\hline Other enterococcus spp. (3) & NT & $3(100 \%)$ & $3(100 \%)$ & $3(100 \%)$ & \\
\hline Staphylococcus aureus (3) & $0(0 \%)$ & $3(100 \%)$ & $3(100 \%)$ & NT & \\
\hline
\end{tabular}

3rd cephalosporin: ceftazidime and ceftriaxone; 4th cephalosporin: cefpirome

$N T$ not test

${ }^{a}$ Excluded the NT episodes

negative microorganisms, colistin (100\% susceptibility rate) is the best choice of antibiotic treatment as shown through our data. For Gram-positive isolates, linezolid resulted in the highest susceptibility rate in this study.

\section{Discussion}

Comorbidities are important risk factors for postoperative complications, mortality, and infections [21-25]. From our study, sex, renal failure, peptic ulcer disease, fluid and electrolyte disorders, acute cholecystitis, and an age greater than 65 years were risk factors for BSI in HBPS patients. In a recent study from Jackson SS [26], a model was developed containing procedure type, age, race, smoking, diabetes, liver disease, obesity, renal failure, and malnutrition. This model showed good discrimination (c-statistic, 0.73 ) and calibration in predicting surgical-site infection, using electronically available comorbidities. Our study identified a predictive risk score model for BSI. According to our model, BSI risk ranged from $1.43 \%$ to $11.95 \%$; AUROC to predict BSI risk was 0.72 (95\% CI 0.69-0.75).

Liver cirrhosis is a very important factor for mortality and morbidity in HBPS. There are 236 liver cirrhosis patients in our series (data not shown). In total, 18 out of
$349(5.6 \%)$ BSI patients were noted with liver cirrhosis and odds ratio is 1.26 (95\% CI $0.75-2.02, P=0.4)$. Eighty-two patients died in our series, and 12 patients were with liver cirrhosis (odds ratio 3.99, 95\% CI 2.13-7.46, $P<0.0001$ ). There is no significant association between liver cirrhosis and BSI, but a significant association was noted with mortality.

Enterobacteriaceae (especially E. coli and K. pneumoniae) were the most commonly isolated microorganisms from bloodstream infections in our HBPS patients. These findings reflect those of many other infectious studies in hepatobiliary or pancreatic diseases [27, 28]. In this study, for Enterobacteriaceae isolated from community-acquired BSI, most microorganisms are sensitive to third- and fourth-generation cephalosporins, imipenem, and colistin, but less sensitive (about 70\%) to quinolone. But for Enterobacteriaceae isolated from healthcare-associated BSI, most microorganisms are less sensitive to antibiotic treatment. Among the microorganisms isolated from healthcare-associated BSI, colistin-resistant E. coli were noted in 5 out of 16 patients (about 31.2\%). In total, 4 out of 5 colistin-resistant $E$. coli bacteremic patients died (data not shown) due to sepsis-induced multiple organ failure. The rising frequency of colistin-resistant $E$. coli is associated with food animals and the spread of the mor- 1 gene 
[29]. The colistin-resistant Enterobacteriaceae may become a serious problem in healthcare-associated BSI for HBPS patients in the future. From our data, we suggest imipenem as the best choice for treatment.

Several limitations of the study should be mentioned. First, this study is a retrospective data analysis with information obtained from an administrative database, which is a potential source of bias. Surgical clinical coding is prone to subjectivity, variability, and error, especially since $13 \%$ of the data underwent changes to the primary diagnosis and $12 \%$ to the primary procedure [30]. Secondly, this study was done by a single hospital only, without external validation. Therefore, the study results do not reflect the whole picture of BSI in HBPS patients nationwide or globally. Thirdly, HBPS consists of many different and complex procedures, and it is very difficult to classify the risk factors for BSI after HBPS.

\section{Conclusion}

Dividing patients into low-, intermediate-, and high-risk groups on the basis of sex, age, status of comorbidity (renal failure, peptic ulcer disease, fluid and electrolyte disorders, and acute cholecystitis), a predictive BSI risk score model was developed. We hope that the results from this study can propel physicians to set up new prophylactic protocols for high-risk patients (determined by their comorbidities) who receive HBPS. If bloodstream infections unfortunately occur, physicians can refer to the results of this study to decide the appropriate antibiotics to administer, especially given the increased prevalence of certain types of antibiotic-resistant bacteria in recent years. This is an occurrence worthy of global attention and further action.

\begin{abstract}
Acknowledgements We thank Janice C. Yang (from the Massachusetts Institute of Technology, MA, USA) for her critical English review of the manuscript. We also thank the funding support for research and publication from MacKay Medical College and MacKay Memorial Hospital, Taiwan (107DMH0100277).
\end{abstract}

\section{Compliance with ethical standards}

Conflict of interest The authors declare that they have no conflict of interest.

Open Access This article is distributed under the terms of the Creative Commons Attribution 4.0 International License (http://crea tivecommons.org/licenses/by/4.0/), which permits unrestricted use, distribution, and reproduction in any medium, provided you give appropriate credit to the original author(s) and the source, provide a link to the Creative Commons license, and indicate if changes were made.

\section{References}

1. Mayo SC, Shore AD, Nathan $\mathrm{H}$ et al (2010) National trends in the management and survival of surgically managed gallbladder adenocarcinoma over 15 years: a population-based analysis. J Gastrointest Surg 14:1578-1591

2. Mayo SC, Shore AD, Nathan H et al (2011) Refining the definition of perioperative mortality following hepatectomy using death within 90 days as the standard criterion. HPB (Oxford) 13:473-482

3. Nathan H, Segev DL, Mayo SC et al (2012) National trends in surgical procedures for hepatocellular carcinoma: 1998-2008. Cancer 118:1838-1844

4. Mayo SC, Gilson MM, Herman JM et al (2012) Management of patients with pancreatic adenocarcinoma: national trends in patient selection, operative management, and use of adjuvant therapy. J Am Coll Surg 214:33-45

5. Mayo SC, Heckman JE, Shore AD et al (2011) Shifting trends in liver-directed management of patients with colorectal liver metastasis: a population-based analysis. Surgery 150:204-216

6. Sato M, Tateishi R, Yasunaga $\mathrm{H}$ et al (2012) Mortality and morbidity of hepatectomy, radiofrequency ablation, and embolization for hepatocellular carcinoma: a national survey of 54,145 patients. J Gastroenterol 47:1125-1133

7. Kimura W, Miyata H, Gotoh M et al (2014) A pancreaticoduodenectomy risk model derived from 8575 cases from a national single-race population (Japanese) using a web-based data entry system: the 30-day and in-hospital mortality rates for pancreaticoduodenectomy. Ann Surg 259:773-780

8. Morikane K (2017) Epidemiology and risk factors associated with surgical site infection after different types of hepatobiliary and pancreatic surgery. Surg Today 47(10):1208-1241

9. Yeh CN, Chen MF, Lee WC et al (2002) Prognostic factors of hepatic resection for hepatocellular carcinoma with cirrhosis: univariate and multivariate analysis. J Surg Oncol 81:195-202

10. Meguro M, Mizuguchi T, Kawamoto M et al (2014) Highest intraoperative lactate level could predict postoperative infectious complications after hepatectomy, reflecting the Pringle maneuver especially in chronic liver disease. J Hepatobiliary Pancreat Sci 21:489-498

11. Ruan DY, Lin ZX, Li Y, Jiang N et al (2015) Poor oncologic outcomes of hepatocellular carcinoma patients with intra-abdominal infection after hepatectomy. World J Gastroenterol 21:5598-5606

12. Dong ZM, Chidi AP, Goswami J et al (2015) Prior inpatient admission increases the risk of post-operative infection in hepatobiliary and pancreatic surgery. HPB (Oxford) 17:1105-1112

13. Yurkovich M, Avina-Zubieta JA, Thomas J et al (2015) A systematic review identifies valid comorbidity indices derived from administrative health data. J Clin Epidemiol 68:3-14

14. Elixhauser A, Steiner C, Harris DR et al (1998) Comorbidity measures for use with administrative data. Med Care 36:8-27

15. Xu Y, Li N, Lu M, Dixon E et al (2017) Comparison of risk adjustment methods in patients with liver disease using electronic medical record data. BMC Gastroenterol 17:5

16. Annane D, Bellissant E, Cavaillon JM (2005) Septic shock. Lancet 365:63-78

17. Garner JS, Jarvis WR, Emori TG et al (1998) CDC definitions for nosocomial infections, 1988. Am J Infect Control 16:128-140

18. Friedman ND, Kaye KS, Stout JE et al (2002) Health care-associated bloodstream infections in adults: a reason to change the accepted definition of community-acquired infections. Ann Intern Med 137:791-797 
19. Yeh GY, McCarthy EP, Wayne PM et al (2011) Tai chi exercise in patients with chronic heart failure: a randomized clinical trial. Arch Intern Med 171:750-757

20. Noh DY, Roh JK, Kim YH et al (2017) Symposium: "Oncology Leadership in Asia". Cancer Res Treat 49:283-291

21. Chok KS, Chan MM, Dai WC et al (2017) Survival outcomes of hepatocellular carcinoma resection with postoperative complications-a propensity-score-matched analysis. Medicine (Baltimore) 96:e6430

22. Yokoo H, Miyata H, Konno H et al (2016) Models predicting the risks of six life-threatening morbidities and bile leakage in 14,970 hepatectomy patients registered in the National Clinical Database of Japan. Medicine (Baltimore) 95:e5466

23. Aoki S, Miyata H, Konno H et al (2017) Risk factors of serious postoperative complications after pancreaticoduodenectomy and risk calculators for predicting postoperative complications: a nationwide study of 17,564 patients in Japan. J Hepatobiliary Pancreat Sci 24:243-251

24. Asano T, Yamada S, Fujii T et al (2017) The Charlson age comorbidity index predicts prognosis in patients with resected pancreatic cancer. Int J Surg 39:169-175

25. Pascual V, Alonso N, Simo M et al (2016) Bloodstream infections caused by Escherichia coli producing AmpC beta-lactamases: epidemiology and clinical features. Eur J Clin Microbiol Infect Dis 35:1997-2003
26. Xuan C, Shamonki JM, Chung A et al (2014) Microbial dysbiosis is associated with human breast cancer. PLoS ONE 9:e83744

27. Toh HS, Chuang YC, Huang CC et al (2012) Antimicrobial susceptibility profiles of Gram-negative bacilli isolated from patients with hepatobiliary infections in Taiwan: results from the Study for Monitoring Antimicrobial Resistance Trends (SMART), 2006-2010. Int J Antimicrob Agents 40(Suppl):S18S23

28. Shigeta H, Nagino M, Kamiya J et al (2002) Bacteremia after hepatectomy: an analysis of a single-center, 10-year experience with 407 patients. Langenbecks Arch Surg 387:117-124

29. Liu YY, Wang Y, Walsh TR et al (2016) Emergence of plasmidmediated colistin resistance mechanism MCR-1 in animals and human beings in China: a microbiological and molecular biological study. Lancet Infect Dis 16:161-168

30. Nouraei SA, Hudovsky A, Frampton AE et al (2015) A study of clinical coding accuracy in surgery: implications for the use of administrative big data for outcomes management. Ann Surg 261:1096-1107

Publisher's Note Springer Nature remains neutral with regard to jurisdictional claims in published maps and institutional affiliations. 